3 Laura Martin-Sancho ${ }^{1}$, Mary K. Lewinski ${ }^{2}$, Lars Pache ${ }^{1}$, Charlotte A. Stoneham ${ }^{2}$, Xin Yin ${ }^{1}$, Dexter 4 Pratt $^{3}$, Christopher Churas ${ }^{3}$, Sara B. Rosenthal ${ }^{3}$, Sophie Liu ${ }^{3}$, Paul D. De Jesus ${ }^{1}$, Alan M. O’Neill ${ }^{4}$, Anshu

5 P. Gounder ${ }^{1}$, Courtney Nguyen ${ }^{1}$, Yuan $\mathrm{Pu}^{1}$, Aaron L. Oom², Lisa Miorin ${ }^{5,6}$, Ariel Rodriguez-Frandsen ${ }^{1}$, 6 Matthew Urbanowski ${ }^{5}$, Megan L. Shaw ${ }^{5,7}$, Max W. Chang ${ }^{3}$, Christopher Benner ${ }^{3}$, Matthew B. Frieman ${ }^{8}$,

\section{Functional Landscape of SARS-CoV-2 Cellular Restriction}

${ }^{1}$ Immunity and Pathogenesis Program, Infectious and Inflammatory Disease Center, Sanford Burnham Prebys Medical Discovery Institute, La Jolla, CA, USA

${ }^{2}$ Department of Medicine, University of California at San Diego, and the VA San Diego Healthcare System, San Diego, California, USA

${ }^{3}$ Department of Medicine, University of California San Diego, La Jolla, CA 92093, USA

${ }^{4}$ Department of Dermatology, University of California San Diego, La Jolla, CA 92093, USA

${ }^{5}$ Department of Microbiology, Icahn School of Medicine at Mount Sinai, New York, NY, USA

${ }^{6}$ Global Health and Emerging Pathogens Institute, Icahn School of Medicine at Mount Sinai, New York, NY, USA

${ }^{7}$ Department of Medical Biosciences, University of the Western Cape, Cape Town, South Africa

${ }^{8}$ Department of Microbiology and Immunology, University of Maryland at Baltimore, Baltimore, Maryland, USA

${ }^{9}$ Department of Medicine, Division of Infectious Diseases, Icahn School of Medicine at Mount Sinai, New York, NY, USA

${ }^{10}$ The Tisch Institute, Icahn School of Medicine at Mount Sinai, New York, NY, USA

${ }^{11}$ Department of Computer Science and Engineering, University of California San Diego, La Jolla, CA 92093, USA

${ }^{12}$ Division of Infectious Diseases, Northwestern University Feinberg School of Medicine, Chicago, IL, 60611, USA

\footnotetext{
* Corresponding author:
}

Sumit K. Chanda

Sanford Burnham Prebys Medical Discovery Institute 10901 North Torrey Pines Road, La Jolla, CA 92037, USA

E-mail: schanda@sbpdiscovery.org

Tel.: +1 (858)795-5241

Fax: +1 (858)795-5225 


\section{SUMMARY}

43

A deficient interferon response to SARS-CoV-2 infection has been implicated as a determinant of severe COVID-19. To identify the molecular effectors that govern interferon control of SARS-CoV2 infection, we conducted a large-scale gain-of-function analysis that evaluated the impact of human interferon stimulated genes (ISGs) on viral replication. A limited subset of ISGs were found to control viral infection, including endosomal factors that inhibited viral entry, nucleic acid binding proteins that suppressed viral RNA synthesis, and a highly enriched cluster of ER and Golgi-resident ISGs that inhibited viral translation and egress. These included the type II integral membrane protein BST2/tetherin, which was found to impede viral release, and is targeted for immune evasion by SARSCoV-2 Orf7a protein. Overall, these data define the molecular basis of early innate immune control of viral infection, which will facilitate the understanding of host determinants that impact disease severity and offer potential therapeutic strategies for COVID-19.

KEYWORDS: SARS-CoV-2, innate immunity, interferon, ISG, BST2, Orf7a 


\section{INTRODUCTION}

The ongoing coronavirus disease 2019 (COVID-19) pandemic, caused by severe acute respiratory syndrome coronavirus 2 (SARS-CoV-2), is responsible for a reported 23.5 million infections, and over 800,000 deaths worldwide as of this writing (Dong et al., 2020). Following infection with SARS-CoV2, COVID-19 clinical presentation ranges from asymptomatic or mild (suggested to account for $\sim 80 \%$ of infections), to severe disease that typically requires hospitalization and assisted respiration (Huang et al., 2020). While age and co-morbidities, such as obesity and cardiovascular disease, have been linked to COVID-19 severity, recent data suggest that cellular immune responses to viral infection are also a critical determinant of disease outcome (Mathew et al., 2020). For instance, loss-of-function mutations in the immune sensor TLR7 and downregulation of the type I interferon (IFN) response have been associated with severe COVID-19 (van der Made et al., 2020). In addition, two recent studies that conducted an integrated immune analysis of COVID-19 patients found impaired IFN responses in severe and critically ill patients (Arunachalam et al., 2020; Hadjadj et al., 2020). Further support for the role of IFN in COVID-19 outcome comes from a study of 127 patients receiving interferon beta-1b in combination with lopinavir-ritonavir and ribavirin, which reported lower SARS-CoV-2 viral load and shedding in the lungs and reduced length of hospitalization (Hung et al., 2020). Taken together, these data underscore an emerging role for IFN-mediated cellular responses in the control of SARS-CoV-2 infection and COVID-19 severity.

Viral infection is sensed by pattern-recognition receptors (PRR), which initiate a signaling cascade that produces cytokines, including IFN. Binding of IFN to its receptor (IFNAR) promotes the transcriptional activation of hundreds of interferon stimulated genes (ISGs), many of which exert antiviral activities (Schoggins et al., 2011). Concerted expression and regulation of these PRRs and downstream signaling molecules, transcription factors, and effectors are necessary to mount a successful antiviral response. Thus, viruses have developed various strategies to interfere with and evade these antiviral programs (García-Sastre, 2017). Recent work has shown that SARS-CoV-2 infection is sensitive to IFN treatment, as RNAseq of COVID-19 patients samples and in vitro infection models revealed upregulation of ISGs (Blanco-Melo et al., 2020; Emanuel et al., 2020; Lamers et al., 2020; Overmyer et al., 2020; Sun et al., 2020). In addition, the ISG LY6E has been identified as a negative regulator of SARS-CoV-2 (Pfaender et al., 2020), and the ISGs AXIN2, CH25H, EPSTI1, GBP5, IFIH1, IFITM2, and IFITM3 were found to block entry of a pseudotyped vesicular stomatitis virus (VSV) harboring SARS-CoV-2 Spike (S) protein (Zang et al., 2020). Ultimately, a comprehensive evaluation of ISGs that inhibit infection of SARS-CoV-2 will be necessary to understand the cellular control of viral infection and their potential impact on COVID-19 outcome.

To uncover the cellular antiviral response to SARS-CoV-2 infection, we conducted a gain-offunction screen using 399 human ISGs. These data revealed that restriction of SARS-CoV-2 is mediated

114 by a limited subset of 65 ISGs, most of which reside in the ER or Golgi compartments and function to 
regulate endoplasmic reticulum-associated protein degradation (ERAD), lipid membrane composition, and vesicle transport. Among these was BST2, found to inhibit viral egress and to be antagonized by SARS-CoV-2 accessory protein Orf7a to rescue virion release. The identification of the ISG subset that direct the antiviral activity of IFN illuminates the molecular and genetic determinants of early immune regulation that contribute to COVID-19 outcome, and provide attractive specific targets for therapeutic intervention.

\section{RESULTS AND DISCUSSION}

\section{IFN-mediated restriction of SARS-CoV-2 relies on a limited subset of ISGs}

To define the cellular effectors that act to limit SARS-CoV-2 infection, we first sought to determine which genes are activated upon IFN stimulation (hereafter referred to as ISGs) in diseaserelevant cell types. Human tracheobronchial epithelial (HTBE) and human alveolar epithelial A549 cells were treated with IFN for $8 \mathrm{~h}$ and then subjected to RNAseq. Using cut-off criteria of $\log 2 \mathrm{FC}>1.5$ and $\mathrm{p}$ value $<0.05$, we identified 139 ISGs upregulated in HTBE, 121 ISGs upregulated in A549 cells, and 152 ISGs upregulated in both HTBE and A549 (Fig S1A). This dataset encompassed ISGs with previously characterized broad-acting antiviral activities that included MX1,OAS1, OASL and IFI6 (Hubel et al., 2019). In addition, Schoggins et al. previously assembled a list of 387 curated ISGs, of which 149 overlapped with the HTBE/A549 dataset (Fig S1B) (Schoggins et al., 2011). We combined these experimental and published datasets, and identified 399 ISGs as available, validated, and fullsequence length cDNA clones (Fig S1B, Table S1A, B).

Next, we evaluated the ability of these 399 ISGs to inhibit SARS-CoV-2 replication using ectopic expression screening. These studies were conducted using the human epithelial cell line 293T, as these cells can be transfected with high efficiencies and support productive replication of SARSCoV-2 when expressing the viral entry factors ACE2 and TMPRSS2 (Hoffmann et al., 2020). 293T cells were transfected with individual ISGs along with ACE2 and TMPRSS 2 for $30 \mathrm{~h}$, and then challenged with SARS-CoV-2 at a low multiplicity of infection $(\mathrm{MOI}=0.0625)$. Cells were fixed at $40 \mathrm{~h}$ postinfection, and infectivity was determined using immunostaining for SARS-CoV-2 nucleoprotein (N) (Fig 1A). cDNA encoding chloramphenicol acetyltransferase (CAT) was included on each plate as 144 negative control, and cDNA encoding the SARS-CoV-2 negative regulator LY6E (Pfaender et al., 2020)

145 was included as positive control (Fig 1A, B). Screens were conducted in duplicate and showed good 146 reproducibility with a Pearson correlation coefficient $(\mathrm{r})=0.81$ (Fig 1C). After applying cut-off criteria 147 for infectivity ( $\log 2 \mathrm{FC}$ at least four standard deviations lower than the CAT negative control) and cell 148 viability (at least 70\% number of cells of the negative control), we identified 65 ISGs that inhibited 149 SARS-CoV-2 replication (Fig 1B). Cross-comparison of these 65 factors with published datasets of 150 upregulated genes from COVID-19 patient samples and in vitro infected lung cell models revealed a 151 significant overlap (Fig S1C), suggesting that these factors are also stimulated in response to SARS- 
152 CoV-2 infection (Blanco-Melo et al., 2020; Emanuel et al., 2020; Overmyer et al., 2020; Sun et al., 153 2020).

154

To further validate the antiviral activity of the ISGs identified in this high-throughput screen, we generated stable cell lines expressing each of these 65 ISGs and assessed their ability to inhibit SARS-CoV-2 replication. Upon transduction of 293T-ACE2 cells with lentiviruses carrying these 65 factors, 7 cell lines did not survive antibiotic selection, so stable lines could only be generated for the remaining 58 ISGs. Next, the ability of these ISG-expressing cells to support replication of SARS-CoV2 was evaluated. Of these, 37 lines showed statistically significant reductions of SARS-CoV-2 replication compared to parental cells ( $\log 2 \mathrm{FC}$ at least four standard deviations lower than parental cells and $\mathrm{p}$ value $\leq 0.05)($ Fig 1 D, E).

Importantly, this screening approach captured both upstream regulators as well as downstream effectors of the IFN response, including the signaling adaptor MYD88, signal transducers STATI and STAT2, transcription factors ELF1, REC8, and ETV6, and several IFN effectors including BST2, IFITM2, and IFITM3, which likely harbor direct antiviral activities. The full list of identified ISGs and their activities are shown in Table $\mathbf{S 2}$ and $\mathbf{S 3}$.

\section{Network model of SARS-CoV-2 antiviral effectors}

ISGs are a heterogenous group of genes with encoded functions ranging from inflammatory pathway signaling to intracellular trafficking, energy metabolism, and nuclear transport (Schoggins, 2019). To better understand the biochemical and functional context by which these 65 ISGs exert antiviral activities, we conducted a supervised network propagation leveraging high confidence proteinprotein interactions and hierarchical relationships (Fig 2, see Methods). Using this analysis, we identified densely interconnected protein clusters that are significantly associated with cellular biological processes (Raudvere et al., 2019). As expected, we found strong association to pathways that stimulate IFN signaling, including cytosolic pattern recognition receptors and regulators of STAT phosphorylation, as well as pathways linked to the type I IFN response, the cellular response to viral infection, and cytokine signaling (Fig 2A, blue boxes). We also observed an enrichment of RNA helicases, and regulators of cell death. Within this group were $D D X 60$, which exhibits antiviral activity against hepatitis C virus (HCV) and VSV (Schoggins et al., 2011), ZBP1, which was recently identified as a sensor of influenza A virus Z-RNA motifs, and $M K L K$, a ZBP1 binding partner and downstream activator of necroptosis in response of viral infection (Fig 2B) (Zhang et al., 2020). Additional enriched clusters included regulation of transport at the Golgi network or the ER (Fig 2C, D), nucleotide metabolism, and regulators of sphingolipid metabolism, including the ISGs B4GALT5 and ST3GAL4 (Fig 2E). Additional ER/Golgi resident factors identified as potent restrictors of SARS-CoV-2 replication included the apolipropotein APOL2 and RSAD2/Viperin, which are involved in lipid 
synthesis and mobilization. This suggests that regulation of the membrane composition at sites relevant for viral replication or trafficking is likely a critical host strategy for the control of SARS-CoV-2 replication. Overall, this network analysis underscores the diversity of activities that underlie the cellular antiviral response to SARS-CoV-2 replication.

\section{Restriction of SARS-CoV-2 entry}

To understand how these antiviral effectors impact viral replication, a selected subset of ISGs were tested for their ability to inhibit specific stages of the SARS-CoV-2 infectious cycle. Firstly, we adopted a pseudotyped VSV expressing SARS-CoV-2 S protein (VSV-S-luciferase) to measure viral entry (Fig 3A, diagram). Then we assessed viral RNA replication by measuring viral RNA at $8 \mathrm{~h}$ postinfection (Fig 3B). Lastly, we infected naïve cells with viral supernatants that were collected at $18 \mathrm{~h}$ post infection to assess late stage activity, encompassing viral translation and egress (Fig 3C). These experimental data were integrated with available bioinformatic resources that provided information on subcellular localization and known function to establish a predictive map of the impact of these ISGs on the SARS-CoV-2 infectious cycle (Fig 4).

Entry of SARS-CoV-2 into the host cell is facilitated by viral S protein binding to the ACE2 cellular receptor promoting endocytosis. Upon entry, SARS-CoV-2 viral particles escape the endosome to initiate viral replication (Hoffmann et al., 2020). Six ISGs reduced entry of the pseudotyped VSV-S by more than 50\%, including LY6E, CLEC4D, UBD, ELF1, FAM46C and REC8 (Fig 3A, 4). LY6E was previously demonstrated to restrict SARS-CoV-2 entry by inhibiting viral $\mathrm{S}$ protein fusion at the membrane (Pfaender et al., 2020). CLEC4D is an integral membrane protein that acts as an endocytic receptor and has been linked to inhibition of bacteria uptake (Wilson et al., 2015). Another ISG affecting viral entry was $U B D / F A T 10$, which is recruited to the incoming Salmonella-containing vacuole (SCV) together with the autophagy cargo receptor p62 (Spinnenhirn et al., 2014), and these serve as signals for lysosomal targeting and pathogen clearance. Since these ISGs have been reported to impact endolysosomal function, it is possible that they interfere with SARS-CoV-2 entry by impeding low pH-

216 dependent entry or endosomal escape. Finally, the transcription factor ELF1 was also found to affect

217 viral entry. ELF1 governs a complex transcriptional program of over 300 genes that are largely distinct

218 from those induced by IFN, suggesting that a secondary antiviral transcriptional cascade acts to inhibit SARS-CoV-2 entry, and potentially other stages of the viral life cycle (Seifert et al., 2019).

\section{Cellular inhibition of SARS-CoV-2 RNA replication}

Following SARS-CoV-2 release into the cytosol, expression of the replicase gene from the viral genomic RNA generates non-structural proteins (nsp). These nsps coordinate the assembly of the replicase-transcriptase complex (RTC) at the ER, which enables viral RNA replication and protein 
replication ( $>50 \%$ inhibition) (Fig 3B), including IFIT3, SPATS2L, DNAJC6, RGSS2, LOC152225, as well as $Z B P 1$ and $B 4 G A L T 5$, which were found to be core components of the cell death and sphingolipid metabolism networks shown in Fig 2B, E.

The IFIT-family includes five members (IFIT1, IFIT1B, IFIT2, IFIT3, and IFIT5), which prevent active viral RNA replication by detection and sequestering of single-stranded 5'-ppp or 2'Ounmethylated RNA (Metz et al., 2013). In this study, we identified three members of this family, IFIT1, IFIT3, and IFIT5, to inhibit SARS-CoV-2 replication, suggesting this family plays an important role in the restriction of SARS-CoV-2. RNA replication was also reduced by the RNA binding protein SPAT2SL. Following stress stimuli, SPAT2SL is recruited to cytoplasmic stress granules, where viral RNA can be sequestered to reduce viral genome synthesis (Miller, 2011; Zhu et al., 2008). Finally, the ISG DNAJC6, a member of the heat shock protein 40 (HSP40) family, was also determined to impact the SARS-CoV-2 replicative stage (Fig 3B). HSP4O family members are known to play critical roles in protein transport, folding, and structural disassembly, and can bind the 3' untranslated region of the mouse hepatitis virus (MHV) coronavirus (Nanda et al., 2003; Rosenzweig et al., 2019). Overall, these data suggest that molecular recognition and targeting of viral RNA is a critical host defense strategy used to interfere with SARS-CoV-2 genome synthesis.

\section{ER- and Golgi resident ISGs inhibit late stage SARS-CoV-2 replication}

Transcription and translation of SARS-CoV-2 subgenomic mRNAs at the ER membrane generate accessory, as well as the structural proteins $\mathrm{S}$, envelope (E), membrane (M), and nucleocapsid (N). S, E, and M are then inserted into the ER and transit through the secretory pathway to commence viral assembly in the ER-Golgi intermediate compartment (ERGIC). Specifically, M, S and E associate with viral genomes encapsidated by the $\mathrm{N}$ protein to form virions that bud from the ERGIC. Virions traffic in vesicles through the trans-Golgi network and are subsequently released by exocytosis. Notably, we found that a majority of ISGs in our assay $(16 / 35,55 \%)$ restricted late stages of viral replication (Fig 3C). Based on their reported function, late stage ISGs were clustered with predicted impacts on translation, ERAD, and vesicle trafficking.

Translation. The 5'-nucleotidase family member $N T 5 C 3$, as well as the broad spectrum antiviral ISG2O, impacted translation or egress of SARS-CoV-2. While reported activities include regulation of nucleotide pools and RNA degradation, both factors have also been implicated in the inhibition of viral translation. Specifically, NT5C3 was found to inhibit translation of HCV proteins, and ISG20 was shown

259 to interfere with translation of VSV by discriminating between self and non-self mRNAs (Metz et al., 260 2013; Wu et al., 2019). 
ERAD. Accumulation of viral proteins during virion assembly at the ER-Golgi interface can trigger ERAD. Accordingly, we found the ERAD regulator ERLINI to strongly attenuate late stages of SARSCoV-2 replication. Two additional factors, RETREGI and FNDC4, also involved in this pathway with roles as a ER-phagy receptor and association with the aggresome (Wilkinson, 2019), were also found to restrict SARS-CoV-2 replication, suggesting that ERAD is a critical cellular antiviral mechanism triggered during SARS-CoV-2 infection.

Vesicle Trafficking. Trans-Golgi vesicle budding was found as an enriched network for the control of SARS-CoV-2 replication (Fig 2C). Proteins within this network include the heat shock protein HSPA8, and the 2',3'-cyclic nucleotide 3' phosphodiesterase $C N P$; both mapped to late stage viral replication (Fig 3C). HSPA8 is involved in vesicle uncoating, whereas $C N P$ was reported to inhibit release of human immunodeficiency virus 1 (HIV-1) (Wilson et al., 2012). Notably, these ISGs were found in a protein complex with $N A P A$, another identified restriction factor for SARS-CoV-2, and a member of the SNARE complex that functions to dock and fuse vesicles to target membranes. Finally, the GTPase $R a b 27 a$ also impeded late stages of replication. Rab27a controls exocytic transport through fusion of multivesicular endosomes to the plasma membrane (Ostrowski et al., 2010), further underscoring the control of vesicular trafficking as a critical antiviral mechanism to control SARS-CoV-2 replication.

\section{BST2 inhibits release of SARS-CoV-2 and is antagonized by Orf7a}

The bone marrow stromal antigen 2 (BST2; also known as CD317 or tetherin) was identified as a potent inhibitor of SARS-CoV-2 replication (Fig 3C). BST2 traffics through the ER and Golgi, and localizes at the plasma membrane and in endosomes. It has been shown to inhibit viral release of several enveloped viruses, including HIV-1, human coronavirus 229E, and SARS-CoV-1, that either bud at the plasma membrane or at the ERGIC by tethering their virions to the cell surface or intracellular membranes (Neil et al., 2008; Taylor et al., 2015; Van Damme et al., 2008; Wang et al., 2014).

BST2 restriction of SARS-CoV-2 replication was further confirmed in ACE2/TMPRSS2expressing $293 \mathrm{~T}$ and Huh7 cells at 24 and 48 h post-infection (Fig 5A, B, S2A). We next conducted loss-of-function studies in HeLa cells, since these harbor constitutive expression of BST2 (Neil et al., 2008; Van Damme et al., 2008), and found that cells depleted for BST2 released significantly more infectious viruses over time (Fig 5C, S2B). Overall, these data strongly support a role for BST2 in the restriction of SARS-CoV-2 replication.

Notably, BST2 expression reduced SARS-CoV-2 RNA replication (53\% reduction compared to control cells) followed by a more potent reduction of viral release ( $74 \%$ reduction) (Fig 3B, C). To further characterize the impact of BST2 on late stage replication, we evaluated viral egress in the 
RNA replication (Siu et al., 2008). We confirmed that this system can recapitulate virus egress, as transfection of viral M, N, and E, but not E and N alone, resulted in secreted N protein (Fig S2C). Using this system, we detected a strong reduction of VLP release in the presence of BST2, evidenced by loss of secreted N, corroborating that BST2 acts to inhibit egress of SARS-CoV-2 (Fig 5D). We next investigated if BST2 colocalizes with SARS-CoV-2 structural proteins. Notably, we detected colocalization of BST2 and structural proteins $\mathrm{M}$ and $\mathrm{S}$ at intracellular foci within the perinuclear region (caption 2, Fig 5E, S2D) but not at the plasma membrane (caption 1, Fig 5E). Together, these data indicate that BST2 spatially associates with SARS-CoV-2 structural proteins during viral assembly and trafficking.

Several viruses have developed evasion strategies to overcome restriction by BST2, including the HIV-1 accessory protein Vpu and SARS-CoV-1 Orf7a (Neil et al., 2008; Taylor et al., 2015; Van Damme et al., 2008). Notably, we found that both HIV-1 Vpu and SARS-CoV-2 Orf7a expression partially rescued BST2-mediated inhibition of SARS-CoV-2 release (Fig 5D), and that both Orf7a and BST2 were incorporated into the VLP particles (Fig 5D) (Fitzpatrick et al., 2010). We further investigated the location of BST2 and Orf7a in the cell and observed that BST2 and Orf7a colocalized in the perinuclear region (Fig 5F). To further investigate Orf7a antagonism of BST2, we infected parental or BST2 $293 \mathrm{~T}$ stable cells with either WT SARS-CoV-2 or a recombinant SARS-CoV-2 that was engineered to replace Orf7a with nanoluciferase (dOrf7a) (kindly provided by Ralph Baric) (Hou et al., 2020). While WT and dOrf7a viruses grew similarly in parental cells, the replication of dOrf7a virus was significantly attenuated in BST2-expressing cells at $48 \mathrm{~h}$ post-infection (Fig 5G). Overall, these data establish BST2 as a potent inhibitor of SARS-CoV-2 egress, and demonstrate that viral Orf7a protein enables immune evasion through the antagonism of BST2 restriction.

\section{Comparative antiviral activities of SARS-CoV-2 restriction factors}

Finally, to understand if discrete cellular defense strategies are deployed to inhibit SARS-CoV2 replication, the restriction dataset was cross-referenced with published single ISG overexpression studies that covered 20 different RNA and DNA viruses, including influenza A virus (FluAV), West Nile virus (WNV), HCV, and HIV-1 (Schoggins et al., 2011, 2014) (Fig 6). Interestingly, ten SARSCoV-2 ISGs were found to reduce replication of four or more viruses (Fig 6). These include well described IFN signaling transducers, signaling molecules, and innate immune sensors STAT2, and MYD88, inhibitors of viral entry IFITM2 and IFITM3 (Brass et al., 2009), and viral nucleic acid binders ZBPI and IFIT1. Conversely, a cluster of 8 ISGs harbored selective activities for SARS-CoV-2 (Fig 6), including ER-Golgi resident proteins NAPA, APOL2, and ERLIN1. Notably, significant enrichment in ISGs that regulate ER homeostasis and Golgi transport suggest that these organelles are critical sites for

334 the cellular control of SARS-CoV-2 replication. Surprisingly, many of these antiviral factors have not

335 been reported to impact other viruses that rely on these membraned compartments for replication and 
assembly, including flavi-, toga-, arteri-, and bunyaviruses, suggesting that these cellular defense mechanisms target unique aspects of coronavirus translation, assembly, and egress.

Taken together, this comprehensive analysis of the ISGs that act to impede SARS-CoV-2 revealed that the IFN response to SARS-CoV-2 infection relies on a limited subset of ISGs that govern a diverse set of cellular functions, including endocytosis, nucleotide biosynthesis and sphingolipid metabolism. Further dissection of these critical host-pathogen interactions, as well as potential viral evasion strategies, will enable insights into the molecular determinants of innate immune control of SARS-CoV-2 replication and clinical disease outcomes.

\section{DATA AVAILABILITY}

The A549 and HTBE RNA-seq data used in this study have been deposited in the Gene Expression

349 Omnibus (GEO) database repository under the accession number GSE156295 (token whyhqquupvadjgn).

\section{ACKNOWLEDGMENTS}

We would like to thank Ralph Baric for providing the dOrf7a SARS-CoV-2, Thomas Rogers for providing the HeLa-ACE2 cells, Kwok-Yung Yuen for providing the rabbit-anti-SARS-CoV-2 N antibody, and Stefan Pohlmann for providing the mammalian expression vector encoding SARS-CoV2 S, pCG1-CoV2-S-HA. We also would like to thank Marisol Chacon for administrative support, Sylvie Blondelle and Larry Adelman for biosafety support, and Rowland Eaden for shipping assistance. We would also like to thank the Viral Vector Core Facility at the SBP for the cDNA normalization and the lentivirus production. This work was supported by the following grants to the Sanford Burnham Prebys Medical Discovery Institute and the Icahn School of medicine at Mount Sinai: DoD: W81XWH-20-10270; DHIPC: U19 AI118610; Fluomics/NOSI: U19 AI135972. This work was also supported by generous philanthropic donations from Dinah Ruch and Susan \& James Blair, from the JPB Foundation,

364 the Open Philanthropy Project (research grant 2020-215611 (5384)) and anonymous donors. Additional 365 support has been provided by DARPA grant HR0011-19-2-0020 and by CRIP (Center for research on 366 Influenza Pathogenesis), a NIAID-funded Center of Excellence for Influenza Research and Surveillance 367 (CEIRS, contract \# HHSN272201400008C). This work was additionally supported by the following 368 grants to Northwestern University Feinberg School of Medicine: a CTSA supplement to NCATS: UL1 369 TR002389; a CTSA supplement to NUCATS with the generous support of the Dixon family: UL1 370 TR001422; and a Cancer Center supplement: P30 CA060553, and the following grant to JG at UC San 371 Diego: NIH grant R37AI081668. This work was also supported by a generous grant from the James B.

372 Pendleton Charitable Trust. The funding sources had no role in the study design, data collection, 
analysis, interpretation, or writing of the report. The content of this study is solely the responsibility of the authors and does not necessarily represent the official views of the National Institutes of Health.

\section{AUTHOR CONTRIBUTIONS}

L.M.-S., M.K.L., C.A.S., J.G. and S.K.C., conceived and designed the experiments. L.M.-S., M.K.L., X.Y., C.A.S., A.P.G., P.D.J, C.N., Y.P., and A.L.O., conducted and/or analyzed the experiments. L.P. and A.M.O. conducted data analysis and representation. A.R.F., M.U., M.C. and C.B. performed and/or analyzed the RNAseq experiments. D.P., C.C., S.L., B.R, and T.I. generated the network model. L.M. and J.F.H. generated essential reagents. L.M.-S., and S.K.C. wrote the manuscript with contributions from all authors. Funding Acquisition, L.P., C.B., A.G.-S., and S.K.C.

\section{DECLARATION OF INTERESTS}

The authors declare no competing interests.

\section{REFERENCES}

Arunachalam, P.S., Wimmers, F., Mok, C.K.P., Perera, R.A.P.M., Scott, M., Hagan, T., Sigal, N., Feng, Y., Bristow, L., Tak-Yin Tsang, O., et al. (2020). Systems biological assessment of immunity to mild versus severe COVID-19 infection in humans. Science eabc6261.

Blanco-Melo, D., Nilsson-Payant, B.E., Liu, W.-C., Uhl, S., Hoagland, D., Møller, R., Jordan, T.X., Oishi, K., Panis, M., Sachs, D., et al. (2020). Imbalanced Host Response to SARS-CoV-2 Drives Development of COVID-19. Cell 181, 1036-1045.e9.

Brass, A.L., Huang, I.-C., Benita, Y., John, S.P., Krishnan, M.N., Feeley, E.M., Ryan, B.J., Weyer, J.L., van der Weyden, L., Fikrig, E., et al. (2009). The IFITM Proteins Mediate Cellular Resistance to Influenza A H1N1 Virus, West Nile Virus, and Dengue Virus. Cell 139, 1243-1254.

Carlin, D.E., Demchak, B., Pratt, D., Sage, E., and Ideker, T. (2017). Network propagation in the cytoscape cyberinfrastructure. PLOS Comput. Biol. 13, e1005598.

Dong, E., Du, H., and Gardner, L. (2020). An interactive web-based dashboard to track COVID-19 in real time. Lancet Infect. Dis. S1473309920301201.

Emanuel, W., Kirstin, M., Vedran, F., Asija, D., Theresa, G.L., Roberto, A., Filippos, K., David, K., Salah, A., Christopher, B., et al. (2020). Bulk and single-cell gene expression profiling of SARS-CoV2 infected human cell lines identifies molecular targets for therapeutic intervention. BioRxiv 2020.05.05.079194.

Fehr, A.R., and Perlman, S. (2015). Coronaviruses: an overview of their replication and pathogenesis. Methods Mol. Biol. Clifton NJ 1282, 1-23.

Fitzpatrick, K., Skasko, M., Deerinck, T.J., Crum, J., Ellisman, M.H., and Guatelli, J. (2010). Direct Restriction of Virus Release and Incorporation of the Interferon-Induced Protein BST-2 into HIV-1 Particles. PLOS Pathog. 6, e1000701.

García-Sastre, A. (2017). Ten Strategies of Interferon Evasion by Viruses. Cell Host Microbe 22, 176184. 
Gordon, D.E., Jang, G.M., Bouhaddou, M., Xu, J., Obernier, K., O’Meara, M.J., Guo, J.Z., Swaney, D.L., Tummino, T.A., Hüttenhain, R., et al. (2020). A SARS-CoV-2-Human Protein-Protein Interaction Map Reveals Drug Targets and Potential Drug-Repurposing (Systems Biology). responses in severe COVID-19 patients. Science eabc6027.

Hoffmann, M., Kleine-Weber, H., Schroeder, S., Krüger, N., Herrler, T., Erichsen, S., Schiergens, T.S., Herrler, G., Wu, N.-H., Nitsche, A., et al. (2020). SARS-CoV-2 Cell Entry Depends on ACE2 and TMPRSS2 and Is Blocked by a Clinically Proven Protease Inhibitor. Cell 181, 271-280.e8.

Hou, Y.J., Okuda, K., Edwards, C.E., Martinez, D.R., Asakura, T., Dinnon, K.H., Kato, T., Lee, R.E., Yount, B.L., Mascenik, T.M., et al. (2020). SARS-CoV-2 Reverse Genetics Reveals a Variable Infection Gradient in the Respiratory Tract. Cell 182, 429-446.e14.

Huang, C., Wang, Y., Li, X., Ren, L., Zhao, J., Hu, Y., Zhang, L., Fan, G., Xu, J., Gu, X., et al. (2020). Clinical features of patients infected with 2019 novel coronavirus in Wuhan, China. Lancet Lond. Engl. 395, 497-506.

Hubel, P., Urban, C., Bergant, V., Schneider, W.M., Knauer, B., Stukalov, A., Scaturro, P., Mann, A., Brunotte, L., Hoffmann, H.H., et al. (2019). A protein-interaction network of interferon-stimulated genes extends the innate immune system landscape. Nat. Immunol. 20, 493-502.

Hultquist, J.F., Hiatt, J., Schumann, K., McGregor, M.J., Roth, T.L., Haas, P., Doudna, J.A., Marson, A., and Krogan, N.J. (2019). CRISPR-Cas9 genome engineering of primary CD4+ T cells for the interrogation of HIV-host factor interactions. Nat. Protoc. 14, 1-27.

Hung, I.F.-N., Lung, K.-C., Tso, E.Y.-K., Liu, R., Chung, T.W.-H., Chu, M.-Y., Ng, Y.-Y., Lo, J., ribavirin in the treatment of patients admitted to hospital with COVID-19: an open-label, randomised, phase 2 trial. Lancet Lond. Engl. 395, 1695-1704.

Lamers, M.M., Beumer, J., van der Vaart, J., Knoops, K., Puschhof, J., Breugem, T.I., Ravelli, R.B.G., human gut enterocytes. Science 369, 50-54.

van der Made, C.I., Simons, A., Schuurs-Hoeijmakers, J., van den Heuvel, G., Mantere, T., Kersten, 441 S., van Deuren, R.C., Steehouwer, M., van Reijmersdal, S.V., Jaeger, M., et al. (2020). Presence of 442 Genetic Variants Among Young Men With Severe COVID-19. JAMA.

443 Mathew, D., Giles, J.R., Baxter, A.E., Oldridge, D.A., Greenplate, A.R., Wu, J.E., Alanio, C., Kuripatients reveals distinct immunotypes with therapeutic implications. Science eabc8511.

Metz, P., Reuter, A., Bender, S., and Bartenschlager, R. (2013). Interferon-stimulated genes and their role in controlling hepatitis $\mathrm{C}$ virus. J. Hepatol. 59, 1331-1341.

448 Miller, C.L. (2011). Stress Granules and Virus Replication. Future Virol. 6, 1329-1338. 111. 
Ostrowski, M., Carmo, N.B., Krumeich, S., Fanget, I., Raposo, G., Savina, A., Moita, C.F., Schauer, K., Hume, A.N., Freitas, R.P., et al. (2010). Rab27a and Rab27b control different steps of the exosome secretion pathway. Nat. Cell Biol. 12, 19-30.

Overmyer, K.A., Shishkova, E., Miller, I., Balnis, J., Bernstein, M.N., Peters-Clarke, T.M., Meyer, J.G., Quan, Q., Muehlbauer, L.K., Trujillo, E.A., et al. (2020). Large-scale Multi-omic Analysis of COVID-19 Severity (Infectious Diseases (except HIV/AIDS)).

Pfaender, S., Mar, K.B., Michailidis, E., Kratzel, A., Boys, I.N., V'kovski, P., Fan, W., Kelly, J.N., Hirt, D., Ebert, N., et al. (2020). LY6E impairs coronavirus fusion and confers immune control of viral disease. Nat. Microbiol. 1-10.

Raudvere, U., Kolberg, L., Kuzmin, I., Arak, T., Adler, P., Peterson, H., and Vilo, J. (2019). $\mathrm{g}$ :Profiler: a web server for functional enrichment analysis and conversions of gene lists (2019 update). Nucleic Acids Res. 47, W191-W198.

Rosenzweig, R., Nillegoda, N.B., Mayer, M.P., and Bukau, B. (2019). The Hsp70 chaperone network. Nat. Rev. Mol. Cell Biol. 20, 665-680.

Schoggins, J.W., Wilson, S.J., Panis, M., Murphy, M.Y., Jones, C.T., Bieniasz, P., and Rice, C.M. (2011). A diverse range of gene products are effectors of the type I interferon antiviral response. Nature 472, 481-485.

Schoggins, J.W., MacDuff, D.A., Imanaka, N., Gainey, M.D., Shrestha, B., Eitson, J.L., Mar, K.B., Richardson, R.B., Ratushny, A.V., Litvak, V., et al. (2014). Pan-viral specificity of IFN-induced genes reveals new roles for cGAS in innate immunity. Nature 505, 691-695.

Seifert, L.L., Si, C., Saha, D., Sadic, M., Vries, M. de, Ballentine, S., Briley, A., Wang, G., ValeroJimenez, A.M., Mohamed, A., et al. (2019). The ETS transcription factor ELF1 regulates a broadly antiviral program distinct from the type I interferon response. PLOS Pathog. 15, e1007634.

Shannon, P. (2003). Cytoscape: A Software Environment for Integrated Models of Biomolecular Interaction Networks. Genome Res. 13, 2498-2504.

Siu, Y.L., Teoh, K.T., Lo, J., Chan, C.M., Kien, F., Escriou, N., Tsao, S.W., Nicholls, J.M., Altmeyer, R., Peiris, J.S.M., et al. (2008). The M, E, and N Structural Proteins of the Severe Acute Respiratory Syndrome Coronavirus Are Required for Efficient Assembly, Trafficking, and Release of Virus-Like Particles. J. Virol. 82, 11318-11330.

Spinnenhirn, V., Farhan, H., Basler, M., Aichem, A., Canaan, A., and Groettrup, M. (2014). The ubiquitin-like modifier FAT10 decorates autophagy-targeted Salmonella and contributes to Salmonella resistance in mice. J. Cell Sci. 127, 4883-4893.

Sun, J., Ye, F., Wu, A., Yang, R., Pan, M., Sheng, J., Zhu, W., Mao, L., Wang, M., Huang, B., et al. (2020). Comparative transcriptome analysis reveals the intensive early-stage responses of host cells to SARS-CoV-2 infection. BioRxiv 2020.04.30.071274.

Taylor, J.K., Coleman, C.M., Postel, S., Sisk, J.M., Bernbaum, J.G., Venkataraman, T., Sundberg, E.J., and Frieman, M.B. (2015). Severe Acute Respiratory Syndrome Coronavirus ORF7a Inhibits Bone Marrow Stromal Antigen 2 Virion Tethering through a Novel Mechanism of Glycosylation Interference. J. Virol. 89, 11820-11833.

Van Damme, N., Goff, D., Katsura, C., Jorgenson, R.L., Mitchell, R., Johnson, M.C., Stephens, E.B., and Guatelli, J. (2008). The interferon-induced protein BST-2 restricts HIV-1 release and is downregulated from the cell surface by the viral Vpu protein. Cell Host Microbe 3, 245-252. 
Wang, S.-M., Huang, K.-J., and Wang, C.-T. (2014). BST2/CD317 counteracts human coronavirus 229E productive infection by tethering virions at the cell surface. Virology 449, 287-296.

Whitt, M.A. (2010). Generation of VSV pseudotypes using recombinant $\Delta$ G-VSV for studies on virus entry, identification of entry inhibitors, and immune responses to vaccines. J. Virol. Methods 169, 365-374.

Wilkinson, S. (2019). ER-phagy: shaping up and destressing the endoplasmic reticulum. FEBS J. 286, $2645-2663$.

Wilson, G.J., Marakalala, M.J., Hoving, J.C., van Laarhoven, A., Drummond, R.A., Kerscher, B., Keeton, R., van de Vosse, E., Ottenhoff, T.H.M., Plantinga, T.S., et al. (2015). The C-type lectin receptor CLECSF8/CLEC4D is a key component of anti-mycobacterial immunity. Cell Host Microbe $17,252-259$.

Wilson, S.J., Schoggins, J.W., Zang, T., Kutluay, S.B., Jouvenet, N., Alim, M.A., Bitzegeio, J., Rice, C.M., and Bieniasz, P.D. (2012). Inhibition of HIV-1 Particle Assembly by 2',3'-Cyclic-Nucleotide 3'Phosphodiesterase. Cell Host Microbe 12, 585-597.

Wu, N., Nguyen, X.-N., Wang, L., Appourchaux, R., Zhang, C., Panthu, B., Gruffat, H., Journo, C., Alais, S., Qin, J., et al. (2019). The interferon stimulated gene 20 protein (ISG20) is an innate defense antiviral factor that discriminates self versus non-self translation. PLOS Pathog. 15, e1008093. membrane fusion. BioRxiv 2020.06.08.141077.

519 Zhu, C.-H., Kim, J., Shay, J.W., and Wright, W.E. (2008). SGNP: an essential Stress

Granule/Nucleolar Protein potentially involved in 5.8s rRNA processing/transport. PloS One 3, e3716.

\section{METHODS}

Cells and Viruses. SARS-CoV-2 USA-WA1/2020, isolated from an oropharyngeal swab from a patient with a respiratory illness who developed clinical disease (COVID-19) in January 2020 in Washington, USA, was obtained from BEI Resources (NR-52281). The recombinant dOrf7a SARS-CoV-2 was kindly provided by Ralph Baric (Hou et al., 2020). These viruses were propagated using Vero E6 cells, collected after one passage, aliquoted, and stored at $-80{ }^{\circ} \mathrm{C}$. Plaque forming unit (PFU) assays were performed to titrate the cultured virus. All experiments involving live SARS-CoV-2 followed the approved standard operating procedures of the Biosafety Level 3 facility at the Sanford Burnham Prebys Medical Discovery Institute. Vero E6 (ATCC CRL-1586), HEK293T (ATCC CRL-3216), HeLa (ATCC

532 CRL-1586), and Huh7 (Apath LLC, Brooklyn) cells were maintained in cell growth media: Dulbecco's 533 modified eagle medium (DMEM, Gibco) supplemented with $10 \%$ heat-inactivated fetal bovine serum 534 (FBS, Gibco), $50 \mathrm{U} / \mathrm{mL}$ penicillin - $50 \mu \mathrm{g} / \mathrm{mL}$ streptomycin (Fisher Scientific), $1 \mathrm{mM}$ sodium pyruvate 535 (Gibco), $10 \mathrm{mM}$ 4-(2-hydroxyethyl)-1-piperazineethanesulfonic acid (HEPES, Gibco), and 1X MEM 
non-essential amino acids solution (Gibco). BHK-21/WI-2 cells (Kerafast, MA) were maintained in DMEM (Gibco) supplemented with $10 \%$ heat-inactivated FBS (Gibco) and $50 \mathrm{U} / \mathrm{mL}$ penicillin $50 \mu \mathrm{g} / \mathrm{mL}$ streptomycin. Human tracheobronchial epithelial (HTBE) cells (ATCC PCS-300-010) were cultured in commercially available airway epithelial cell basal medium following manufacturer's protocol (ATCC). HTBE cells were derived from one donor and all tissues used for isolation of these cells were obtained under informed consent and conform to HIPAA standards to protect the privacy of the donors' personal health information. HEK293T and HeLa cells stably expressing ACE2 (293TACE2/HeLa-ACE2) were generated by transducing HEK293T or HeLa cells with human ACE2expressing lentiviruses, followed by selection of resistant cells with puromycin (InvivoGen) at $2 \mu \mathrm{g} / \mathrm{ml}$ for 14 days. The resistant cells were then maintained in cell growth media supplemented with $1 \mu \mathrm{g} / \mathrm{ml}$ puromycin. ACE2 expression was confirmed by western blot analysis. All cells were tested and were confirmed to be free of mycoplasma contamination.

Antibodies. The antibodies used in this study include: Immunofluorescence: rabbit-anti-SARS-CoV-2 $\mathrm{N}$ antibody (gift from Kwok-Yung Yuen, University of Hong Kong), mouse anti-HM1.24 (BST2) (a gift from Chugai Pharmaceutical Co., Kanagawa, Japan), rat anti-FLAG-AlexaFluor-488 (Biolegend, \#637317), mouse anti-HA-AlexaFluor-594 (Biolegend, \#901511), donkey anti-mouse-AlexaFluor-488 (Jackson ImmunoResearch, \#715-545-150), donkey anti-mouse-Rhodamine-Red-X (Jackson ImmunoResearch, \#715-295-150). Western blotting: rabbit polyclonal anti-BST2 (NIH AIDS Reagent Program, Division of AIDS, NIAID, NIH: Anti-BST-2 Polyclonal (cat\# 11721) from Drs. Klaus Strebel and Amy Andrew), mouse monoclonal anti-V5 tag (Invitrogen, \#R960-25), mouse monoclonal antiGAPDH (GeneTex, \#GTX627408), mouse monoclonal anti-FLAG M2 (Sigma, \#F1804), rabbit monoclonal anti- $\beta$-actin antibody (Cell Signaling, \#4970) and rabbit monoclonal anti-CoxIV antibody (Cell Signaling \#4850).

Plasmids. Lentiviral constructs: pLX304 constructs for each of the ISGs, and GFP and CAT as negative controls were obtained from the ORFeome library. psPAX2 (Addgene, \#12260), and pMD2.G (Addgene, \#12259). SARS-CoV-2 contructs: dsDNA gene fragments (gBlocks) encoding human-codonoptimized SARS-CoV-2 proteins M, M-FLAG, E, E-V5, N, N-V5, and Orf7a N- or C-terminally tagged with 3xFLAG tag, corresponding to the SARS-CoV-2 Wuhan-Hu-1 isolate (genbank MN908947.3), were synthesized by Integrated DNA Technologies (IDT). The gene fragments were inserted into the pcDNA3.1(-) backbone between NotI and EcoRI restriction sites using an In-fusion seamless cloning strategy (Takara Bio). The mammalian expression vector encoding COV2 S, pCG1-COV2-S-HA, was obtained from Prof. Stefan Pohlmann (Infection Biology Unit, German Primate Center - Leibniz Institute for Primate Research, University Göttingen (Hoffmann et al., 2020)). 
RNA-seq experiments. HTBE and A549 cells were seeded overnight and then treated with $100 \mathrm{IU} / \mathrm{ml}$ universal interferon beta (IFN, R\&D Systems), or left untreated. At $8 \mathrm{~h}$ post-treatment, cell were lysed in Trizol (Thermo Fisher) and RNA was extracted using RNeasy Mini Kit (Qiagen). Strand-specific ribosomal RNA-depleted sequencing libraries were produced according to standard Illumina protocols, and sequencing was carried out on an Illumina HiSeq 2500. The human hg38 reference genome and RefSeq gene annotation were used for spliced read alignment and gene assignment. Experiments were conducted in duplicate and 412 genes were defined as ISGs based on $\log 2 \mathrm{FC}>1.5$ and $\mathrm{p}$ value $<0.05$.

Overexpression cDNA screen. A targeted overexpression cDNA screen was carried out in human epithelial cells to identify ISGs that restrict the replication of SARS-CoV-2. 399 ISGs were selected for this gain-of-function screen based on experimental, published data, and availability as full-length, sequence-validated cDNA clones. These cDNAs were hand-picked from the ORFeome collection, which contains $\sim 17,000$ full-length, sequenced, V5-epitope tagged human ORFs in the lentiviral expression vector pLX304. Each of these 399 cDNAs were individually arrayed in 384-well plates at a concentration of 40ng/well along with human ACE2 and TMPRSS2 (10 ng), and $0.25 \mu \mathrm{l}$ of the transfection reagent Fugene 6 (Promega). After 20 min incubation at room temperature, 3,000 293T cells diluted in cell growth media (see cells and viruses section) were seeded per well and incubated at $37^{\circ} \mathrm{C}, 5 \% \mathrm{CO}_{2}$. At $30 \mathrm{~h}$ post-transfection, cells were mock-treated or infected with SARS-CoV-2 (USAWA1/2020) at a MOI 0.0625 for $40 \mathrm{~h}$ at $37^{\circ} \mathrm{C}, 5 \% \mathrm{CO}_{2}$. Cells were then fixed with $5 \%$ PFA (Boston BioProducts) for 4 hours at room temperature and then washed twice with 1xPBS. Cells were permeabilized with $0.5 \%$ Triton X-100 for $20 \mathrm{~min}$, followed by two washes with 1xPBS and blocking with 3\% BSA (Sigma) for $1 \mathrm{~h}$ at room temperature. Anti-SARS-CoV-2 N rabbit serum was added for 1 $\mathrm{h}$ at room temperature, followed by three washes with 1xPBS and a 1-h incubation with Alexa Fluor 568-conjugated anti-rabbit secondary antibody (Thermo Fisher Scientific) diluted in 3\% BSA. Following three washes with PBS, cells were stained with DAPI (4,6-diamidine-2-phenylindole, KPL), and plates were sealed and stored at $4^{\circ} \mathrm{C}$ until imaging.

High-content imaging and data analysis. Viral replication was assessed using high-throughput 600 microscopy. The assay plates were imaged using the IC200 imaging system (Vala Sciences) located at 601 the Conrad Prebys Center for Chemical Genomics (CPCCG). The analysis software Columbus v2.5 602 (Perkin Elmer) was used to calculate infectivity (number Alexa 568+ objects/number DAPI+ objects). 603 Screens were run in duplicate and the infectivity values for each well were normalized to the median of 604 the negative control CAT, and used to calculate the $\log 2 \mathrm{FC}$. The hit calling strategy was based on $605 \log 2 \mathrm{FC}$. Factors with a corresponding $\log 2 \mathrm{FC}<4 *$ Stdev CAT, and cell viability $>70 \%$ CAT were 606 considered restriction factors. 
Generation lentivirus and 293T-ACE2-ISG/GFP cells. Lentiviruses were generated for each of the 65 ISGs that were found to restrict SARS-CoV-2 replication. Briefly, 293T cells at passage 10 were cultured in monolayer on matrigel-coated plates. After reaching $90 \%$ of density, three plasmids, including pLX304-ISG/GFP, psPAX2 (Addgene), and pMD2.G (Addgene), were co-transfected into cells at a ratio of 3:2:1 using PEI (VWR). After $16 \mathrm{~h}$ incubation, transfection media were replaced with fresh DMEM supplemented with 10\% FBS. Viral supernatants were collected at $48 \mathrm{~h}$ post-transfection with an estimated transduction unit of $2 \times 10^{4}$ lentiviral particles. Lentiviruses were used to transduce 293T-ACE2 cells $(\mathrm{MOI}=3$ ) pre-treated with $10 \mu \mathrm{g} / \mathrm{ml}$ Polybrene (Life Technologies), followed by selection of resistant cells with Blasticidin (InvivoGen) at $10 \mu \mathrm{g} / \mathrm{ml}$ for 14 days. 293T-ACE2-ISG/GFP resistant cells were maintained in cell growth media supplemented with $2 \mu \mathrm{g} / \mathrm{ml}$ Blasticidin.

Network analyses. To understand the biochemical and functional context by which the identified antiviral ISGs function, we explored a network-based approach that could integrate these ISGs ("seed" proteins) with existing knowledge. Towards this aim, we used a pipeline that employs a combination of scripts and Cytoscape applications. First, to explore the highest confidence interactions of "seed" proteins, we selected the STRING - Human Protein Links - High Confidence (Score $>=0.7$ ) proteinprotein interaction network available on NDEx as the "background" network (link provided below). We then performed network propagation to select a neighborhood of 343 proteins ranked highest by the algorithm with respect to these seeds (Carlin et al., 2017). This "neighborhood" network (including all edges among the 343 proteins) was extracted from the background network. We then identified densely interconnected regions, i.e. "communities" within the neighborhood network, using the community detection algorithm HiDeF via the Community Detection APplication and Service (CDAPS) (manuscript in press, app available at http://apps.cytoscape.org/apps/cycommunitydetection). The result of HiDeF from CDAPS was a "hierarchy" network where each node represented a community of proteins, and edges denoted containment of one community (the "child") by another (the "parent"). Finally, the hierarchy network was styled, communities were labeled by functional enrichment using gProfiler (via CDAPS) and a layout was applied. The STRING - Human Protein Links - High

635 Confidence (Score $>=0.7$ ) network is available in the Network Data Exchange (NDEx) at 636 http://ndexbio.org/\#/network/275bd84e-3d18-11e8-a935-0ac135e8bacf.

638 Generation pseudotyped SARS-CoV-2 virus. VSV pseudotyped with spike (S) protein of SARS$639 \mathrm{CoV}-2$ was generated according to a published protocol (Whitt, 2010). Briefly, BHK-21/WI-2 cells 640 (Kerafast, MA) transfected with SARS-CoV-2 S protein were inoculated with VSV-G pseudotyped $\Delta \mathrm{G}-$ 641 luciferase VSV (Kerafast, MA). After a 2 hour incubation at $37{ }^{\circ} \mathrm{C}$, the inoculum was removed and cells 642 were treated with DMEM supplemented with $5 \% \mathrm{FBS}, 50 \mathrm{U} / \mathrm{mL}$ penicillin, and $50 \mu \mathrm{g} / \mathrm{mL}$ 643 streptomycin. Pseudotyped particles were collected $24 \mathrm{~h}$ post-inoculation, then centrifuged at $1,320 \times \mathrm{g}$ 644 to remove cell debris and stored at $-80{ }^{\circ} \mathrm{C}$ until use. 
646 Mapping into SARS-CoV-2 infectious cycle studies. Mapping studies were conducted in parallel 647 using 293T-ACE2-ISG/GFP cells. Briefly, multiple 96-well plates were seeded with 50,000 293T648 ACE2-ISG/GFP cells/well and incubated overnight at $37^{\circ} \mathrm{C}, 5 \% \mathrm{CO}_{2}$. To determine the effect of the identified ISGs on viral entry, 293T-ACE2-ISG/GFP cells were infected with VSV-S-luciferase and incubated for $16 \mathrm{~h}$. The activity of firefly luciferase was then measured using the bright-Glo ${ }^{\mathrm{TM}}$ luciferase assay (Promega) for quantitative determination. To measure RNA replication and late stages, cells were infected with SARS-CoV-2 (USA-WA1/2020) at a MOI 4 for $1 \mathrm{~h}$ on ice. Viral inoculum was removed and cells were washed twice with 1xPBS and supplemented with cell growth media (see cells and viruses section). At $6 \mathrm{~h}$ post-infection, SARS-CoV-2 RNA replication was measured. Briefly, intracellular viral RNA was purified from infected cells using the TurboCapture mRNA Kit (Qiagen) in accordance with the manufacturer's instructions. The purified RNA was subjected to first-strand cDNA synthesis using the high-capacity cDNA reverse transcription kit (Applied Biosystems, Inc). Real-time quantitative PCR (RT-qPCR) analysis was then performed using TaqPath one-step RT-qPCR Master Mix (Applied Biosystems, Inc) and, ActinB CTRL Mix (Applied Biosystems, Inc) for housekeeping genes, and the 660 following primers and probe for qPCR measurements of viral genes: N-Fwd: 5'661 TTACAAACATTGGCCGCAAA-3'; N-Rev: 5'-GCGCGACATTCCGAAGAA-3'; N-Probe: 5'662 FAM-ACAATTTGCCCCCAGCGCTTCAG-BHQ-3'. To evaluate late stages, supernatants collected 663 at $18 \mathrm{~h}$ post-infection were used to infect naïve Vero E6 cells. At $18 \mathrm{~h}$ post-infection cells were then 664 fixed with 5\% PFA (Boston BioProducts) for 4 hours at room temperature and then subjected to 665 immunostaining and imaging for SARS-CoV-2 N protein and DAPI (described in overexpression cDNA screen section).

Generation of CRISPR-Cas9 BST2 KO HeLa-ACE2 cells. Detailed protocols for RNP production have been previously published (Hultquist et al., 2019). Briefly, lyophilized guide RNA (gRNA) and tracrRNA (Dharmacon) were suspended at a concentration of $160 \mu \mathrm{M}$ in $10 \mathrm{mM}$ Tris-HCL, 150mM $\mathrm{KCl}, \mathrm{pH}$ 7.4. $5 \mu \mathrm{L}$ of $160 \mu \mathrm{M}$ gRNA was mixed with $5 \mu \mathrm{L}$ of $160 \mu \mathrm{M}$ tracrRNA and incubated for $30 \mathrm{~min}$

672 at $37^{\circ} \mathrm{C}$. The gRNA:tracrRNA complexes were then mixed gently with $10 \mu \mathrm{L}$ of $40 \mu \mathrm{M}$ Cas9 (UC-

673 Berkeley Macrolab) to form CRISPR-Cas9 ribonucleoproteins (crRNPs). Five $3.5 \mu \mathrm{L}$ aliquots were

674 frozen in Lo-Bind 96-well V-bottom plates (E\&K Scientific) at $-80^{\circ} \mathrm{C}$ until use. BST2 gene was targeted

675 by 5 pooled gRNA derived from the Dharmacon pre-designed Edit-R library for gene knock-out. BST2 676 (g1:TGCATCCAGGGAAGCCATTA, CM-011817-01; g2:TTGGGCCTTCTCTGCATCCA, CM- $677 \quad 011817-02$ g3:TTGAGGAGCTTACCACAGTG, CM-011817-03;

678 TCACTGCCCGAAGGCCGTCC, CM-011817-04; g5: CACCATCAAGGCCAACAGCG, CM679 011817-05). Non-targeting negative control gRNA (Dharmacon, U-007501) was delivered in parallel. 680 Each electroporation reaction consisted of $2.5 \times 10^{\wedge} 5$ HeLa-ACE2 cells, $3.5 \mu \mathrm{L}$ crRNPs, and $20 \mu \mathrm{L}$ 681 electroporation buffer. HeLa-ACE2 cells were grown in fully supplemented MEM (10\% FBS, 
1xPen/Strep, 1x non-essential amino acids) to $70 \%$ confluency, suspended and counted. crRNPs were thawed and allowed to come to room-temperature. Immediately prior to electroporation, cells were centrifuged at $400 \mathrm{xg}$ for 3 minutes, supernatant was removed by aspiration, and the pellet was resuspended in $20 \mu \mathrm{L}$ of room-temperature SE electroporation buffer plus supplement (Lonza) per reaction. $20 \mu \mathrm{L}$ of cell suspension was then gently mixed with each crRNP and aliquoted into a 96-well electroporation cuvette for nucleofection with the 4-D Nucleofector X-Unit (Lonza) using pulse code EO-120. Immediately after electroporation, $80 \mu \mathrm{L}$ of pre-warmed media was added to each well and cells were allowed to rest for 30 minutes in a $37^{\circ} \mathrm{C}$ cell culture incubator. Cells were subsequently moved to 12 -well flat-bottomed culture plates pre-filled with $500 \mu \mathrm{L}$ pre-warmed media. Cells were cultured at $37^{\circ} \mathrm{C} / 5 \% \mathrm{CO} 2$ in a dark, humidified cell culture incubator for 4 days to allow for gene knock-out and protein clearance prior to downstream applications.

SARS-CoV-2 viral growth assays. To evaluate SARS-CoV-2 viral growth, the amount of released infectious particles was measured by plaque assay. Briefly, supernatants from SARS-CoV-2 infected cells were collected at indicated time points and stored at $-80^{\circ} \mathrm{C}$ until used. 600,000 Vero E6 cells were seeded and incubated overnight at $37^{\circ} \mathrm{C} / 5 \% \mathrm{CO} 2$ in 12-well plates. Confluent Vero E6 cells were then washed once with $1 \mathrm{xPBS}$ and infected with $100 \mu 1$ of virus-containing supernatants that were serially diluted 1:10. Plates were incubated $1 \mathrm{~h}$ at room temperature, followed by inoculum removal and addition of $1 \mathrm{ml}$ overlay media (2xMEM and 2.5\% Avicel (FMC BioPolymer, RC-591 NF) at 1:1 ratio). 2xMEM contains $100 \mathrm{ml}$ 10x MEM (Gibco), $10 \mathrm{ml}$ 100x penicillin-streptomycin (Fisher Scientific), $10 \mathrm{ml}$ 100x L-Glutamine, $6 \mathrm{ml} \mathrm{35 \%} \mathrm{BSA,} 10 \mathrm{ml} 10 \mathrm{mM}$ 4-(2-hydroxyethyl)-1-piperazineethanesulfonic acid (HEPES, Gibco), $24 \mathrm{~mL}$ 5\% NaHCO3 (Gibco) and $340 \mathrm{ml}$ water. Plates were incubated 3 days at $37^{\circ} \mathrm{C}$, $5 \% \mathrm{CO} 2$, and then fixed and stained using 0.1\% Crystal Violet and 5\% PFA (Boston BioProducts) overnight at $4^{\circ} \mathrm{C}$.

VLP assays. HEK-293T cells seeded in 6-well plates were transfected using Lipofectamine 2000 (Thermo-Fisher) with $625 \mathrm{ng}$ each of plasmids encoding M-FLAG, E-V5, N-V5 (Fig 5D), or $500 \mathrm{ng}$ of M, E, and N-V5 (Fig S2C), with or without 625 ng 3xFLAG-Orf7a or human codon-optimized HIV$1 \mathrm{Vpu}$ (pVpHu from Klaus Strebel) with or without 75 ng BST2 (pcDNA3.1-BST-2 from Autumn Ruiz and Edward Stephens). After 24 hours, supernatants were collected and clarified of cell debris then pelleted through $20 \%$ sucrose at 23,500 $\mathrm{x}$ g for $1 \mathrm{hr}$ at $4^{\circ} \mathrm{C}$. Pelleted VLPs and cells were lysed in $2 \mathrm{X}$

713 Laemmli SDS-PAGE buffer, then run on 12\% SDS-PAGE gels, transferred to PVDF membranes and 714 blotted with the indicated antibodies.

716 Colocalization studies. Immunofluorescence Staining: $2 \times 10^{4} \mathrm{HeLa}-\mathrm{ACE} 2$ cells were seeded on $12 \mathrm{~mm}$ glass coverslips in 24 -well plates, $24 \mathrm{~h}$ prior to transfection. The cells were transfected with $800 \mathrm{ng}$ total plasmid DNA, using Lipofectamine 2000 (Thermo-Fisher), diluted in Optimem, according to 
manufacturer's instructions. HeLa-ACE2 cells were either transfected with equal amounts (200 ng) of SARS-CoV-2 structural proteins M, E, N, S-HA, or M-FLAG, E, N, and empty plasmid (pcDNA3.1). HeLa-ACE2 cells were also transfected with 800 ng ORF7a-3xFLAG. 24 h post-transfection, cells were washed briefly in $4^{\circ} \mathrm{C}$ PBS before incubation with ice-cold $4 \%$ paraformaldehyde (PFA, diluted in PBS, $\mathrm{pH}$ 7.4). The PFA was allowed to warm to RT as the cells were fixed for 20 minutes, the PFA was removed and cells washed $3 \mathrm{x}$ in 1 XPBS (5 min per wash). The fixed cells were quenched with $50 \mathrm{mM}$ Ammonium chloride (in PBS) for 5 minutes RT, washed $3 \times$ in PBS, and permeabilized with $0.2 \%$ Triton X-100 for 7 minutes (RT). The cells were again washed in three times in 1X PBS before incubation with $2 \%$ bovine serum albumin (BSA) in PBS for 30 minutes, prior to incubation with primary antibodies overnight at $4^{\circ} \mathrm{C}$. Cells transfected with $\mathrm{M}, \mathrm{E}, \mathrm{N}$ and S-HA were stained overnight with mouse anti-HM1.24 (BST-2) antibody (diluted 1:300 in 1\% BSA in PBS) at $4^{\circ} \mathrm{C}$. The following day, the cells were washed 3x PBS and incubated with donkey anti-mouse-AlexaFluor-488 (1:400) for $2 \mathrm{hr}$ RT. The cells were washed 3x PBS (10 min per wash) and blocked with $2 \%$ BSA in PBS supplemented with 5\% normal mouse serum for $1 \mathrm{hr}$ RT, briefly washed in 2\% BSA, and incubated with mouse anti-HA-Alexa-594 (1:200) and 4',6-diamidino-2-phenylindole (DAPI), diluted to $1 \mu \mathrm{g} / \mathrm{ml}$ for 2 hr RT. Cells transfected with M-FLAG, E and N were stained overnight with mouse anti-BST-2 (diluted 1:300 in 1\% BSA in PBS). The following day the cells were washed 3x PBS and incubated with donkey anti-mouse-Rhodamine-Red-X (1:400) for $2 \mathrm{hr}$ RT. The cells were washed 3x PBS (10 min per wash) and blocked with 2\% BSA in PBS supplemented with 5\% normal mouse serum for $1 \mathrm{hr}$ RT, briefly washed in 2\% BSA, and incubated with rat anti-FLAG-Alexa-488 (diluted 1:200) and $1 \mu \mathrm{g} / \mathrm{ml}$ DAPI for $2 \mathrm{hr}$ RT. Cells transfected with Orf7a-3xFLAG were stained overnight with mouse anti- HM1.24 (diluted 1:300). The following day the cells were washed 3x PBS and incubated with donkey antimouse-Rhodamine-Red-X (1:400) for $2 \mathrm{hr}$ RT. The cells were washed 3x PBS (10 min per wash) and blocked with 2\% BSA in PBS supplemented with 5\% normal mouse serum for $1 \mathrm{hr}$ RT, briefly washed in 2\% BSA, and incubated with rat anti-FLAG-Alexa-488 (diluted 1:200) and $1 \mu \mathrm{g} / \mathrm{ml}$ DAPI for $2 \mathrm{hr}$ RT. Following immunostaining, the cells were washed extensively in PBS, and briefly in distilled-water, before mounting in Mowiol (Polyvinyl alcohol) mounting medium (prepared in-house). Microscopy: Images were captured at 100x magnification $(1344 \times 1024$ pixels $)$ using an Olympus IX81 widefield microscope fitted with a Hamamatsu CCD camera. For each field, a Z-series of images was collected,

748 deconvolved using the nearest-neighbor algorithm (Slidebook software V.6, Imaging Innovations, Inc)

749 and presented as Z-stack projections. Inset images are deconvolved single z-section images. Arrow 750 heads indicate areas of colocalization, scale bar $=10 \mu \mathrm{m}$. Image brightness was adjusted using Adobe 751 Photoshop CS3.

\section{FIGURE LEGENDS}


(A) Schematic representation of the gain-of-function screen to identify ISGs that inhibit SARS-CoV-2 replication. (B) Ranked log2FC SARS-CoV-2 infectivity values (blue shading) and normalized cell number (pink shading), after individual overexpression of 399 human ISGs and controls. Dashed lines illustrate cut offs for antiviral ISG hit calling strategy: dotted blue line, infectivity $=4 * \operatorname{Stdev} \log 2 \mathrm{FC}$ $C A T$; dotted pink line, cell viability $=70 \%$ of $C A T$. Controls are shown (CAT, negative; $L Y 6 E$, positive). (C) Correlation plots of $\log 2 \mathrm{FC}$ infectivity values for ISG overexpression screens using 293T cells infected with SARS-CoV-2. $r$ = Pearson correlation coefficient between screens. (D) 293T-ACE2 stably expressing each of the identified ISGs were infected with SARS-CoV-2 (MOI 0.25). At $40 \mathrm{~h}$ postinfection, cells were fixed, stained with DAPI and immunolabelled with anti-SARS-CoV-2 nucleoprotein $(\mathrm{N})$ antibody. $\log 2 \mathrm{FC}$ infectivity was calculated as the percentage of $\mathrm{N}^{+} / \mathrm{DAPI}^{+}$cells relative to parental control wells. Data represent mean \pm SD of three independent experiments $(n=3)$. Statistical significance was calculated using one-way ANOVA with Sidak's multiple comparison posthoc test. Representative images are shown in (E). Scale bar $=10 \mu \mathrm{m}$.

\section{Figure 2 - Network model of SARS-CoV-2 antiviral effectors}

(A) The network containing the 65 identified antiviral ISGs was expanded to include a total of 343 high confidence protein interactors (Score> 0.7 STRING) and subjected to supervised community detection (Carlin et al., 2017; Shannon, 2003). The resultant hierarchy is shown. Here, each node represents a community of densely interconnected proteins, and each edge (arrow) denotes containment of one community (edge target) by another (edge source). Enriched biological processes are indicated. The percentage of each community that corresponds to the 65 antiviral ISGs is shown in dark blue. (B-E) Zoom-in insets from selected protein communities are indicated with an asterisk * in the hierarchy. Nodes indicate proteins, and edges indicate interactions from STRING. Blue nodes indicate ISGs that restricted SARS-CoV-2 replication.

Figure 3 - SARS-CoV-2 antiviral effectors inhibit discrete viral replication steps

782 293T-ACE2 cells stably expressing each of the indicated ISGs were subjected to (A) infection with 783 SARS-CoV-2 pseudotyped VSV luciferase virus (VSV-S-luc) for $16 \mathrm{~h}$ prior to measurement of 784 luciferase signal. In parallel, cells were subjected to synchronized infection with SARS-CoV-2 (MOI = 785 4) for $6 \mathrm{~h}$ prior to measurement of viral RNA (B), or supernatants at $18 \mathrm{~h}$ post-infection were used to 786 infect naïve Vero E6 cells. Infectivity was then determined at $18 \mathrm{~h}$ post-infection using immunostaining 787 for viral $\mathrm{N}$ protein (C). In parallel to these experiments, the impact of these ISGs in 293T-ACE2 cells 788 on SARS-CoV-2 replication at $24 \mathrm{~h}$ post-infection was evaluated (full lifecycle). Results are summarized in the heat map and show the mean $(n=2)$ of relative activities compared to parental cells.

791 Figure 4 - Integrated model of SARS-CoV-2 cellular restriction mechanisms 
ISGs that inhibited SARS-CoV-2 replication were placed at specific positions along the viral infectious cycle based on experimental data generated in Figure 3 in conjunction with Gene Ontology, KEGG, Reactome databases and the literature. Human ISGs are represented in blue, and SARS-CoV-2 proteins in yellow. Asterisks * indicate ISGs that were validated using lentiviral transduction. Dashed lines (edges) represent indirect interactions between these ISGs and the indicated viral proteins based on constitutively expressed interactors of ISGs (Hubel et al., 2019) and reported SARS-CoV-2 interactors (Gordon et al., 2020).

\section{Figure 5 - BST2 inhibits release of SARS-CoV-2 and is antagonized by Orf7a}

801 (A, B) 293T and Huh7 cells transfected with BST2 along with ACE2 and TMPRSS2 were infected with 802 SARS-CoV-2 at the indicated MOIs for $48 \mathrm{~h}$ prior to immunostaining for viral N protein. Shown is 803 quantification of infectivity (\% infected cells) relative to empty vector control (A), and representative 804 images of Huh7 cells infected at MOI 0.03 (B). Data show mean \pm SD from three independent 805 experiments. (C) HeLa-ACE2 parental or BST2 KO cells were infected with SARS-CoV-2 (MOI = 2). 806 At the indicated hours post-infection, supernatants were collected and analyzed by plaque assay in Vero 807 E6 cells. $\mathrm{LoD}=$ limit of detection. Data show mean $\pm \mathrm{SD}$ from one representative experiment in triplicate $808(\mathrm{n}=3)$ of two independent experiments. (D) 293T cells were transfected with M-FLAG, E-V5, N-V5, 809 along with the indicated constructs expressing BST2, human codon-optimized HIV-1 Vpu and/or 810 FLAG-tagged SARS-CoV-2 Orf7a. At $24 \mathrm{~h}$ post-transfection, cell lysates and supernatants (VLPs) were 811 analyzed using SDS-PAGE and immunoblotted with indicated antibodies. (E) HeLa-ACE2 cells 812 transfected with M-FLAG, E, and N, were subjected to immunostaining for BST2 and FLAG, as 813 indicated. Shown are deconvolved widefield microscopic images revealing colocalization of BST2 and $814 \mathrm{M}$ (arrows). Scale bar $=10 \mu \mathrm{m}$. (F) HeLa-ACE2 cells transfected with Orf7a-FLAG were subjected to 815 immunostaining for BST2 and FLAG, as indicated. Shown are confocal images revealing colocalization 816 of BST2 and Orf7a (arrows). Scale bar $=10 \mu \mathrm{m}$. (G) Parental 293T-ACE2 or BST2 stable cells were 817 infected with WT or dOrf7a $(\mathrm{MOI}=1)$. At indicated times post-infection, supernatants were collected 818 and analyzed by plaque assay in VeroE6 cells. Data show mean \pm SD from one representative 819 experiment in triplicate $(n=3)$ of two independent experiments. Statistical significance was calculated 820 using one-way ANOVA with Dunnet's post-hoc (A), Student's t-test (C), or Tukey's multicomparison 821 test $(\mathrm{G})$.

\section{Figure 6 - Comparative antiviral activities of SARS-CoV-2 restriction factors}

824 Heat map showing normalized infection upon overexpression of indicated ISGs across 21 viruses. Data 825 for SARS-CoV-2 were generated within this study. Data for the remaining 20 viruses were obtained 826 from previously published work (Schoggins et al., 2011, 2014). Virus families are indicated. 827 Chikungunya virus (CHIKV), O'nyong'nyong virus (ONNV), Sindbis virus (SINV), Venezuelan equine 828 encephalitis virus (VEEV), Yellow fever virus (YFV), Human parainfluenza virus type 3 (PIV3), 
829 Human metapneumovirus (HMPV), Newcastle disease virus (NDV), Respiratory syncytial virus (RSV),

830 Measles virus (MV), Equine viral arteritis (EVA), Bunyamwera virus (BUNV), poliovirus (PV), 831 coxsackievirus (CBV), vaccinia virus (VV). 
Figure 1

A

(CAT) (LVGE)

399 ISGs individually arrayed

Negative control Positive control

80

0000

0000

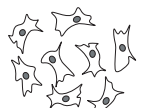

SARS-COV-2

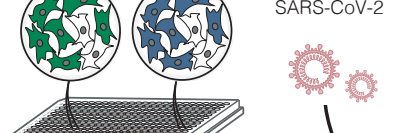

293T cells

$\vec{\longrightarrow}$

INFECTION $\mid 40$ h
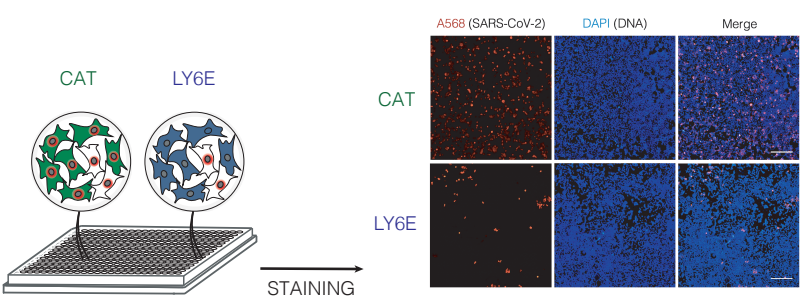

B
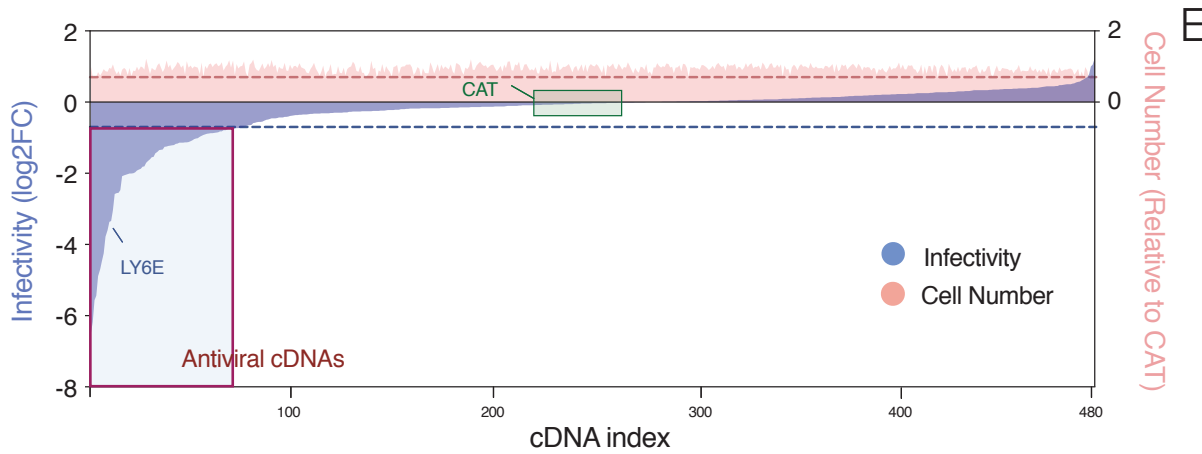

C
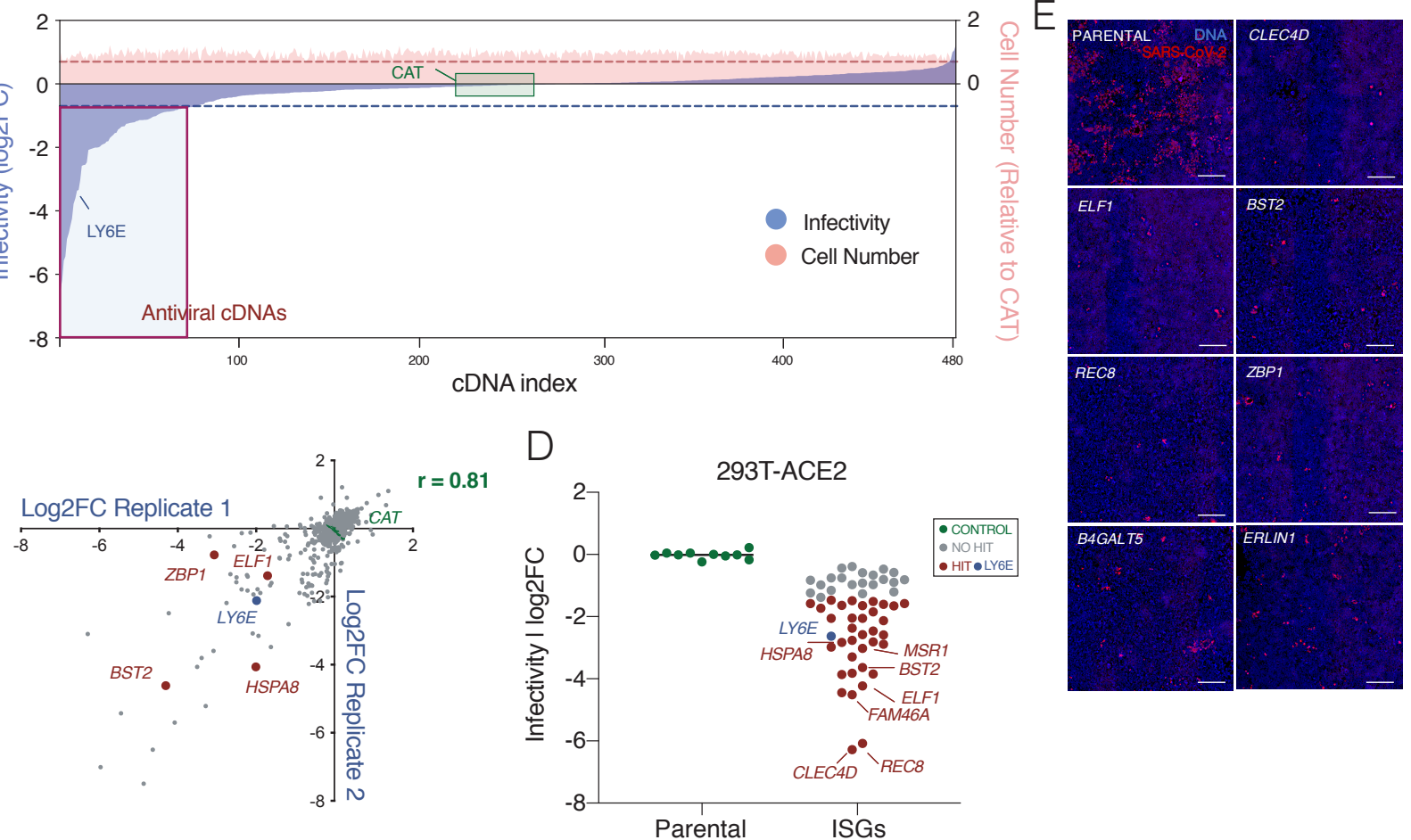
Figure 2

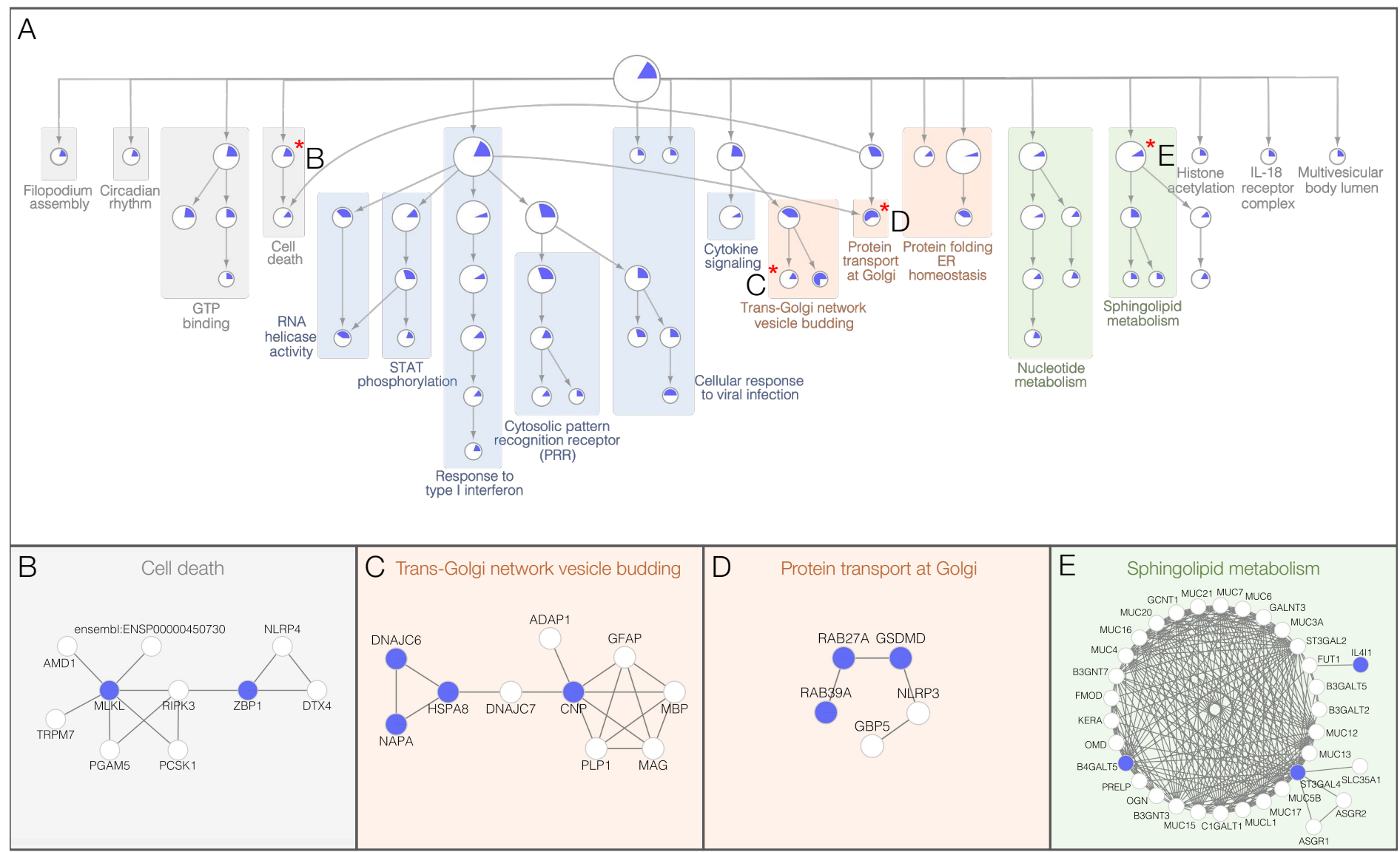


Figure 3

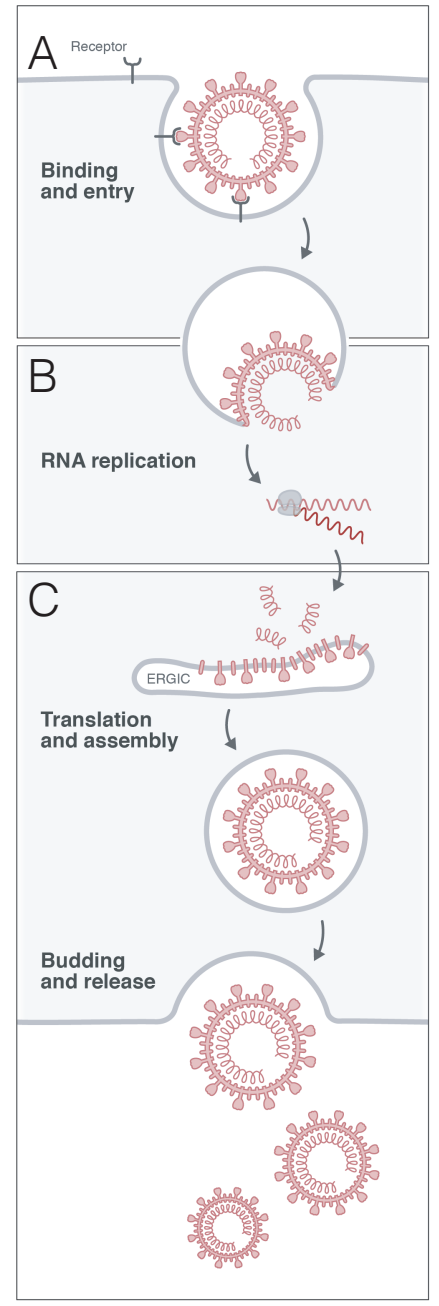

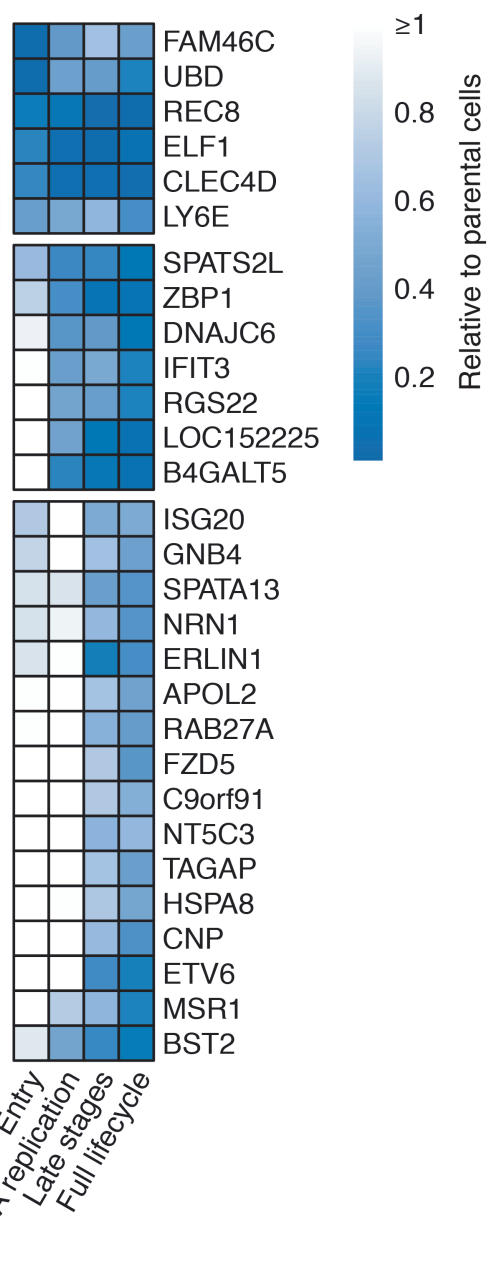


Figure 4

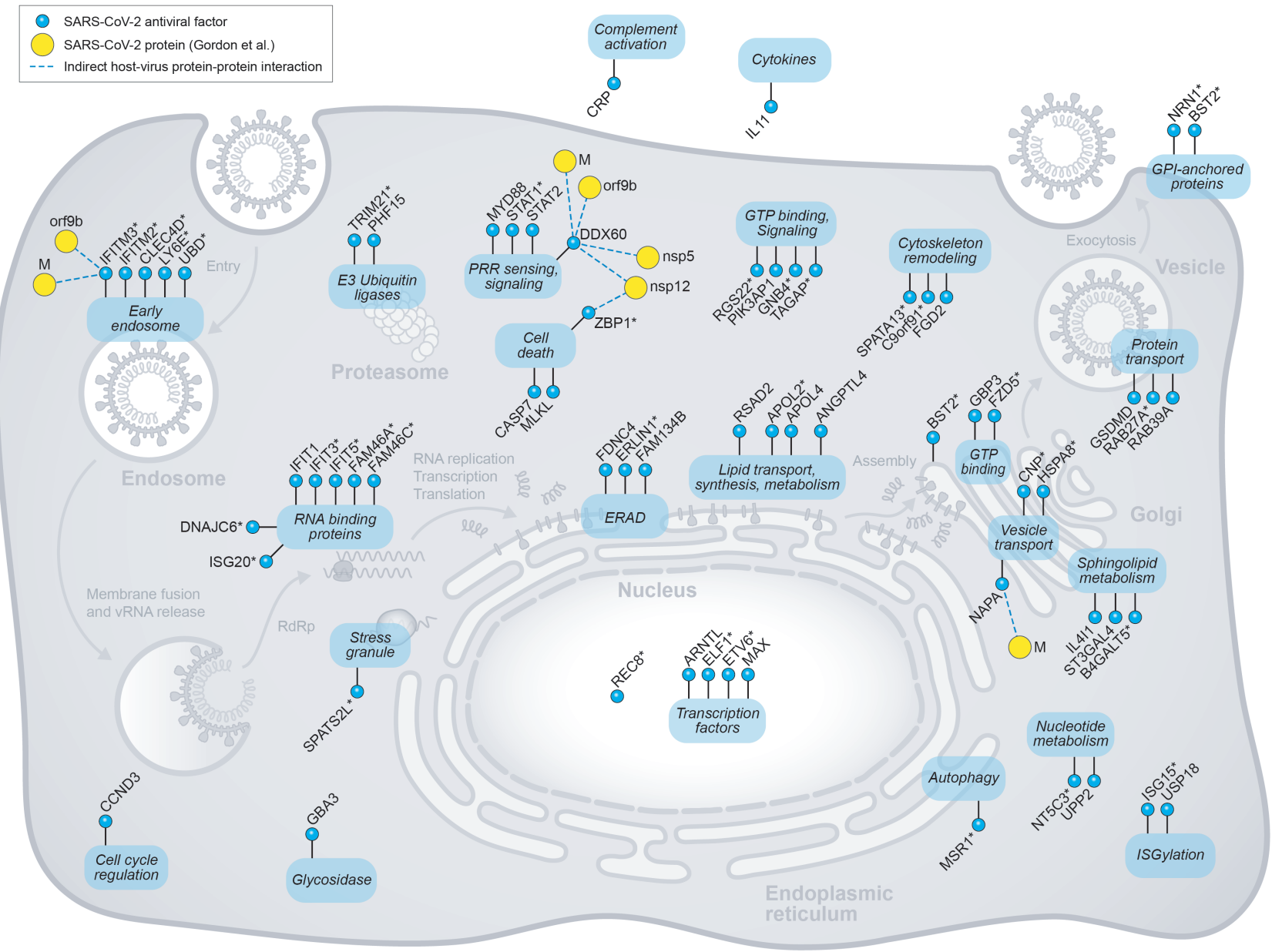


Figure 5
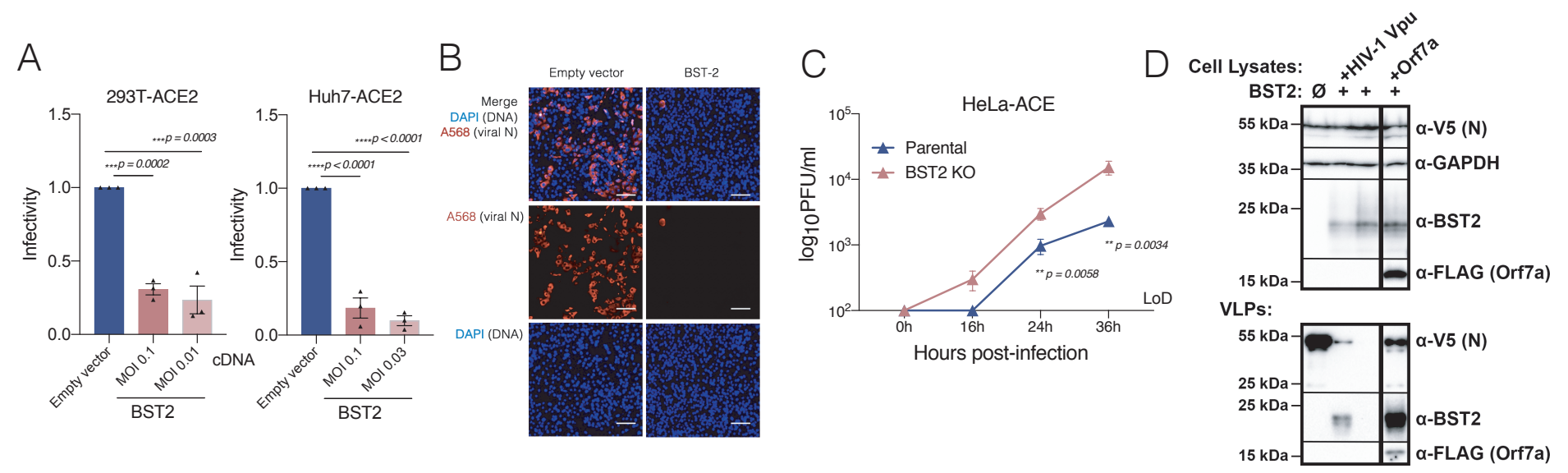

E

M-FLAG, E, N: BST2-Rhx, FLAG-488
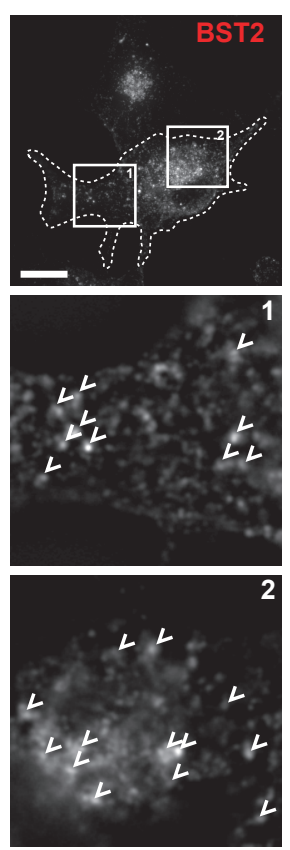
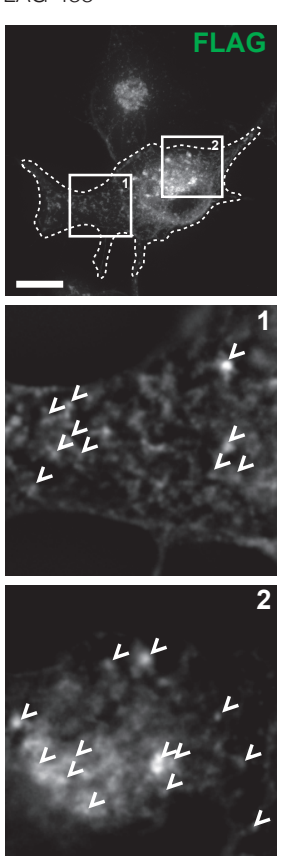
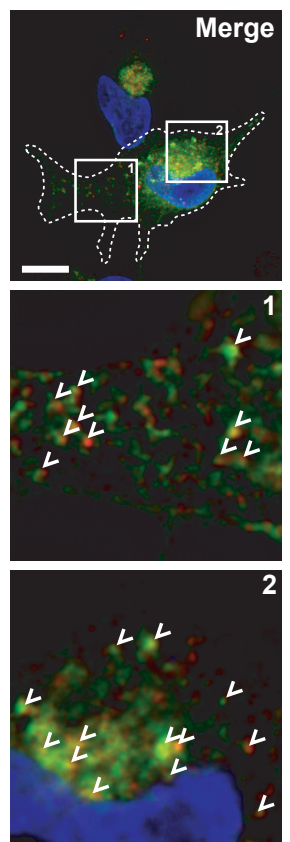

Orf7a-FLAG: BST2-Rhx, FLAG-488
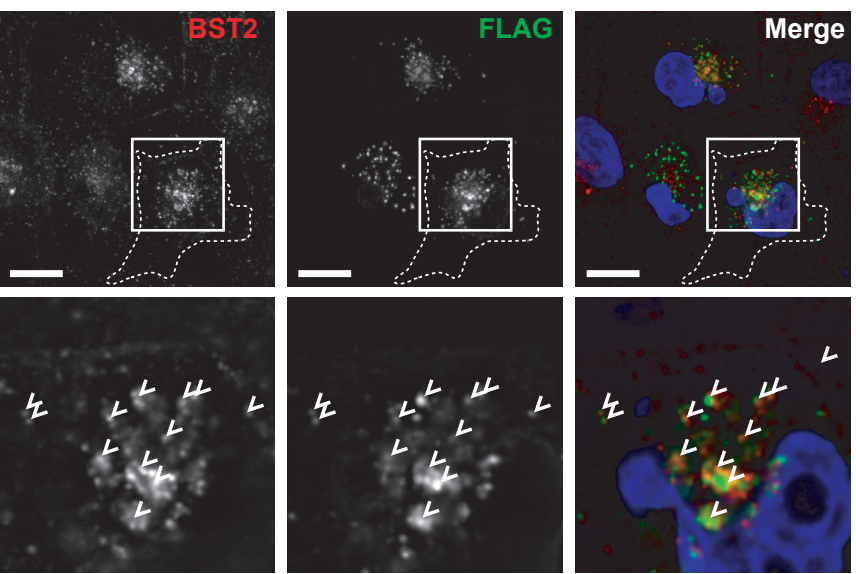

G

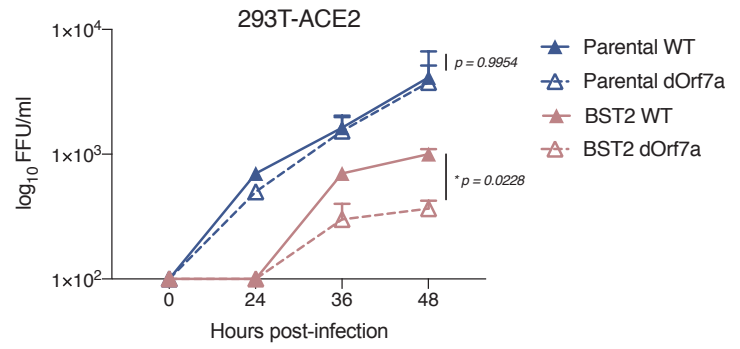


Figure 6

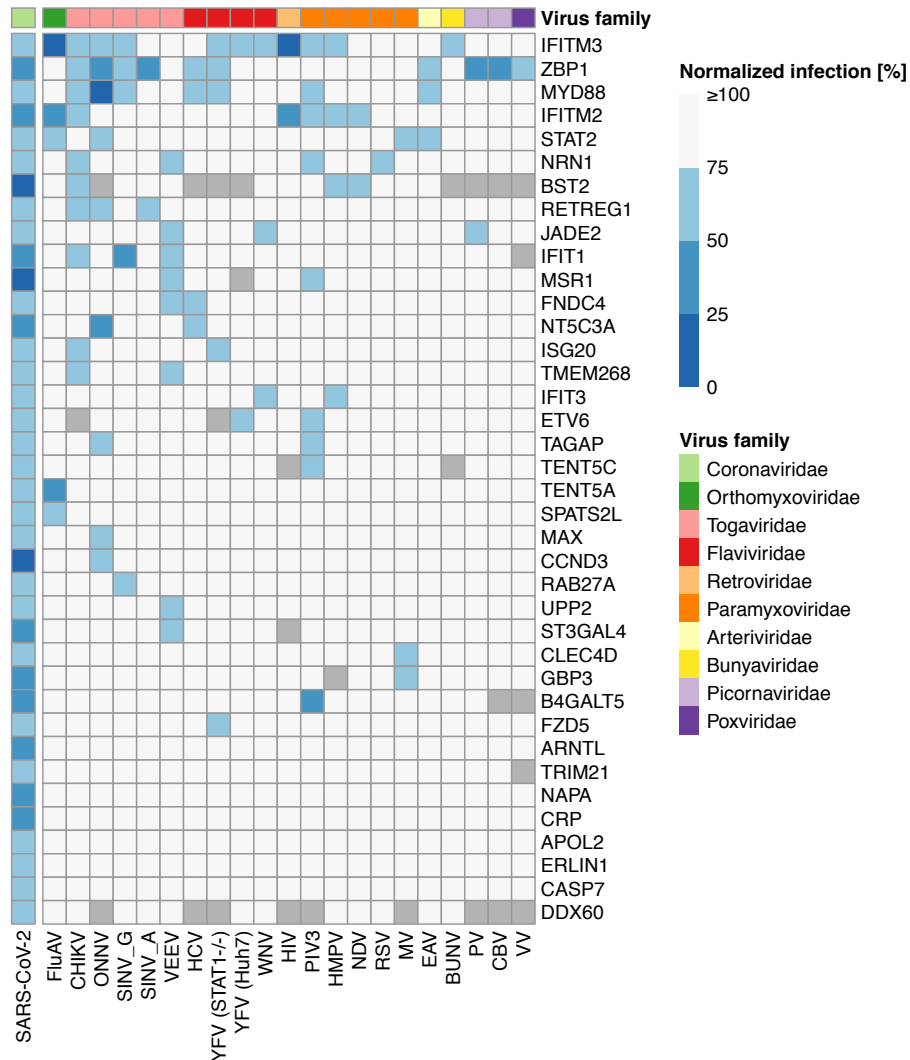

